\title{
Moderate electric fields can inactivate Escherichia coli at room temperature
}

\author{
Luís F. Machado, Ricardo N. Pereira, Rui C. Martins, José A. Teixeira, António A. Vicente* \\ IBB - Institute for Biotechnology and Bioengineering, Centre of Biological Engineering, Universidade do Minho, 4710-057 Braga, Portugal
}

\section{A R T I C L E I N F O}

\section{Article history:}

Received 20 February 2009

Received in revised form 17 July 2009

Accepted 31 August 2009

Available online 4 September 2009

\section{Keywords:}

Moderate electric fields

E. coli

Death kinetics

Non-thermal inactivation

Pasteurization

Sterilization

\begin{abstract}
A B S T R A C T
The inactivation of Escherichia coli using moderate electric fields (MEF) below $25^{\circ} \mathrm{C}$, was investigated. Keeping the temperature always below $25^{\circ} \mathrm{C}$ demonstrated that electric fields are involved in the inactivation of E. coli, without possible synergistic temperature effects. Electric fields above $220 \mathrm{~V} \mathrm{~cm}^{-1}$ promoted death rates of $3 \log _{10}$ cycles of $E$. coli in less than $6 \mathrm{~min}$, and even higher rates at greater electric fields, while presumably overcoming the thermal degradation caused by conventional high temperature treatments. A non-thermal model was proposed that successfully describes the E. coli death kinetics under this treatment. SEM observations of $E$. coli cells after the exposure to the MEF treatment, revealed changes at the cell membrane level, indicating a possible cause for the cell death rates. These results show that this treatment holds potential for sterilization of thermolabile products (e.g. serum and other physiological fluids, food products), by itself or as a complement of the traditional heat-dependent techniques.
\end{abstract}

() 2009 Elsevier Ltd. All rights reserved.

\section{Introduction}

Food industry aims at producing microbiologically safe foods and also at prolonging the shelf life. Generally a thermal treatment is required, since the inactivation of pathogenic and spoilage microorganisms is indispensable. The most common thermal treatments are: cooking (including, e.g. baking, roasting, frying pasteurization and sterilization), that alter the nutritional value and improve food safety; dehydration and drying, that reduce water activity by evaporation, thus extending the shelf life of various foodstuffs (Vicente et al., 2006). These conventional treatments are common practice in food processing in spite of their harmful effects. Flavor ingredients, nutrients and vitamins, which are heat sensitive, are damaged as food components are maintained during a certain time at high temperatures (Rastogi, 2003).

At present consumers demand guaranteed safety and quality from new products, which forces the food industry to search new alternative techniques. Some nonconventional methods (thermal and non-thermal) had been applied to practice. Ohmic heating $(\mathrm{OH})$, known also by the name of moderate electric fields (MEF) processing, is one of the most famous alternative thermal treatments using electric current passed through food. The principal advantage of $\mathrm{OH}$ is the ability to heat materials rapidly and uniformly, including particulated foods (Vicente et al., 2006). If uniform heating occurs throughout a food, process times can be shortened resulting in improved flavor and nutrient retention, leading to a higher quality product both from the nutritional and

\footnotetext{
* Corresponding author. Tel.: +351 253604419; fax: +351 253678986.

E-mail address: avicente@deb.uminho.pt (A.A. Vicente).
}

organoleptic point of view (Machado et al., 2007). The earliest known application of $\mathrm{OH}$ in food processing was the pasteurization of milk in 1919 (Anderson and Finkelstein, 1919). More recently, $\mathrm{OH}$ is being successfully used by the food industry. More than 100 ohmic heaters have been installed, with systems used for whole strawberry and yogurt in Japan and low acid ready-to-eat meals in the USA, among others. The question of whether $\mathrm{OH}$ results in a non-thermal contribution to microbial lethality has been addressed in a number of studies in the literature. Recent studies suggest that a mild electroporation-type mechanism may be at play during $\mathrm{OH}$. The presence of pore-forming mechanisms on vegetable tissue, prokaryotic and eukaryotic cells that are exposed to an electrical field has been confirmed (Sastry et al., 2002). Experiments with beet cubes suggested that diffusion of beet dye increased with electric field strength and decreased with the field frequency, suggesting that electroporation was the mechanism responsible for this phenomenon (Kulshestha and Sastry, 2003). A study on inactivation of Bacillus subtilis spores treated by conventional heating $(\mathrm{CH})$ and $\mathrm{OH}$, at identical temperatures (with the same thermal history), proposed that the inactivation kinetics were accelerated by the applied electric field during ohmic treatment (Cho et al., 1999). The leakage of intracellular constituents of Saccharomyces cerevisiae was found to be enhanced under $\mathrm{OH}$ when compared with $\mathrm{CH}$ in boiling water (Lee and Yoon, 1999).

Pulsed electric fields (PEF) is a new promising non-thermal technology for food pasteurization, resulting in the maintenance of the products quality and extended shelf life. PEF processing involves the application of short pulses $(t<1 \mathrm{~s})$ of high electric field intensity (typically $20-80 \mathrm{kV} \mathrm{cm}^{-1}$ ) (Aronsson et al., 2005). PEF can be used to process liquid and semi-liquid foods. These products 


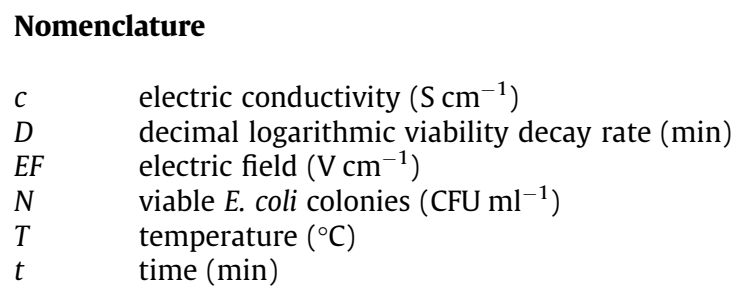

\begin{tabular}{|c|c|}
\hline$W(t)$ & error function (CFU ml ${ }^{-1} \mathrm{~min}^{-1}$ ) \\
\hline$z$ & decimal logarithmic reduction of $D$ value $\left({ }^{\circ} \mathrm{C}\right)$ \\
\hline Subsc & \\
\hline 0 & refers to initial condition \\
\hline ref & refers to the reference variable \\
\hline
\end{tabular}

have to be able to resist high electric fields, have low electric conductivity and no dissolved gas. Successful PEF treatments have been used in food products such as juices, milk, yogurt, soups and liquid eggs (Ho and Mittal, 2000). Some of the most important technical drawbacks or limitations of PEF technologies are the nonavailability of commercial units (high equipment costs), nonuniform treatment, operational and safety problems due to the presence of gas bubbles (Rastogi, 2003).

MEF processing is particularly interesting, because it is far less expensive than PEF, while achieving many of the same effects. MEF is a process characterized by electric field relatively lower (arbitrarily defined between 1 and $1000 \mathrm{~V} \mathrm{~cm}^{-1}$ ) than the ones used for PEF treatments, with the intent to control permeabilization and other non-thermal effects (Sastry et al., 2002; Kulshestha and Sastry, 2003). While the thermal effects of processing food with electric field are widely accepted, the study of non-thermal effects on microorganisms, enzymes and nutrients has been controversial and are not yet fully understood. Regarding the information obtained from the effects of MEF on enzymes and microorganisms (Kulshestha and Sastry, 2003; Cho et al., 1999), it seems highly relevant to verify if the same influence is achieved in the absence of high temperatures, therefore ruling out possible synergistic effects between temperature and electric field.

Herein, we report on the feasibility of MEF for the inactivation of Escherichia coli at temperatures below $25^{\circ} \mathrm{C}$, with the main objectives of: (i) investigating the effect of MEF on the inactivation of $E$. coli; (ii) modeling the death kinetics of $E$. coli when treated with this new non-thermal technique; (iii) validate the MEF technology as an alternative process.

\section{Materials and methods}

\subsection{Sample preparation}

The strain used in this work was E. coli ATCC ${ }^{\circledR} 25922$. The E. coli was obtained as Culti-loop®from Oxoid (Basingstoke, UK) and stored at $4{ }^{\circ} \mathrm{C}$ on slants of MacConkey Agar No. 3 (CM 0115, Oxoid, Basingstoke, UK). E. coli samples were anaerobically cultured in TSB. One milliliter of overnight cultures grown in $100 \mathrm{~mL}$ Erlenmeyer flasks at $35^{\circ} \mathrm{C}$ without agitation were inoculated in $100 \mathrm{~mL}$ of TSB and incubated without agitation for $5 \mathrm{~h}$ (cells in the exponential phase of growth) at $35^{\circ} \mathrm{C}$. The growth phase and the respective parameters (specific growth rate of $2,16 \mathrm{~h}^{-1}$ ) of $E$. coli were determined by taking samples at each 30 min intervals and measuring the absorbance (four replicates) at $595 \mathrm{~nm}$ in a spectrophotometer until the culture reaches the stationary phase. This procedure was repeated three times. For MEF treatments, cells were harvested by centrifugation at $5000 \mathrm{rpm}$ at $4{ }^{\circ} \mathrm{C}, 10 \mathrm{~min}$ (Model 4K15, Sigma, Germany), washed in a phosphate buffer solution ( $\mathrm{pH}=7.0$ and specific electric conductivity of $6.78 \mu \mathrm{S} \mathrm{cm}^{-1}$ at $23^{\circ} \mathrm{C}$ ) and centrifuged again at the mentioned conditions. Cell pellets were finally suspended in $10 \mathrm{~mL}$ phosphate buffer solution. The phosphate buffer was prepared from $0.01 \mathrm{M}$ potassium hydrogen phosphate (Cod. 110, Pronalab, Lisboa, Portugal) and 0.01 M potassium dihydrogen phosphate
(Cod. 8968, José Manuel Vaz Pereira, Lisboa, Portugal) and diluted with ultrapure water from this solution to the final concentration used. Prior to use, the phosphate buffer was autoclaved at $121^{\circ} \mathrm{C}$ for $15 \mathrm{~min}$. This solution was used as the treatment medium. Each cell suspension was kept on a ice bath (maximum $10 \mathrm{~min}$ ) until MEF treatment (Aronsson et al., 2005).

\subsection{Experimental design}

Experiments were performed in a concentrical batch cylindrical glass tube (the "treatment chamber") of $30 \mathrm{~cm}$ total length and an inner diameter of $2.3 \mathrm{~cm}$ with two platinized-titanium electrodes isolated at each edge with Teflon caps (Fig. 1). The sample temperature was controlled by means of a closed circuit cooling system. The cooling system was composed of a temperature-controlled refrigerated bath, using ethylene glycol as cooling agent (Clifton, 64376 NE4-HT, England). A magnetic stirrer was introduced inside the treatment chamber to homogenize the temperature and improve the cooling of the sample. The refrigeration bath temperature was adjusted to keep the sample temperature always below $25^{\circ} \mathrm{C}$. MEF experiments were conducted at electric field values of: $50,160,180,220$, and $280 \mathrm{~V} \mathrm{~cm}^{-1}$, being $E$. coli suspension samples collected, with a micropipette, during the time of the experiment to plot the death curves. Between each experiment, the remaining fluid was removed and the chamber was washed with ethanol at $70 \%$ and rinsed with sterile distilled water. The evolution of the temperature during the experiments was measured with a type-K thermocouple $\left( \pm 1^{\circ} \mathrm{C}\right.$, Omega, 709 , USA $)$ placed at the geometric center of the sample volume and connected to a data acquisition device (National Instruments, USB-9161, USA) working with LabView 7 Express software (National Instruments, NI Datalogger, USA) (Fig. 1(1)).

The distance between the electrodes changed according to the desired value of the electric field. For the experiments at electric field values between 50 and $220 \mathrm{~V} \mathrm{~cm}^{-1}$ a gap between the electrodes of $1-1.4 \mathrm{~cm}$ was used and the supplied voltage ranged from 65 to $234 \mathrm{~V}$. The supplied voltage was controlled through the use of a rheostat (Chuan Hsin, Taiwan) connected to the monophasic alternate current supply system $(231 \mathrm{~V}, 50 \mathrm{~Hz})$ (Fig. 1(3)). For electric field values higher than $220 \mathrm{~V} \mathrm{~cm}^{-1}$ the electrodes were directly connected to a triphase alternate current socket $(400 \mathrm{~V}$, $50 \mathrm{~Hz}$ ). In this case the gap between the electrodes was of $1.4 \mathrm{~cm}$ (Fig. 1(2)). During all the treatments, the electrical field had a sinusoidal waveform and was applied continuously.

The samples were collected at regular time intervals according with each treatment duration. Each treatment duration was selected, case by case, in order to obtain a significant reduction of the microbial load. Once the decay of the number of viable cells is a first order reaction (see results), the duration of the treatment does not alter the cell inactivation rate. The used electric field $\left(\mathrm{V} \mathrm{cm}^{-1}\right)$ was estimated by dividing the potential difference, measured with a digital multimeter $( \pm 1.2 \%$ AC voltage, $\pm 1.5 \%$ AC current, Mastech, MAS-345, China) by the distance between the electrodes, determined externally with a precision Vernier caliper. 


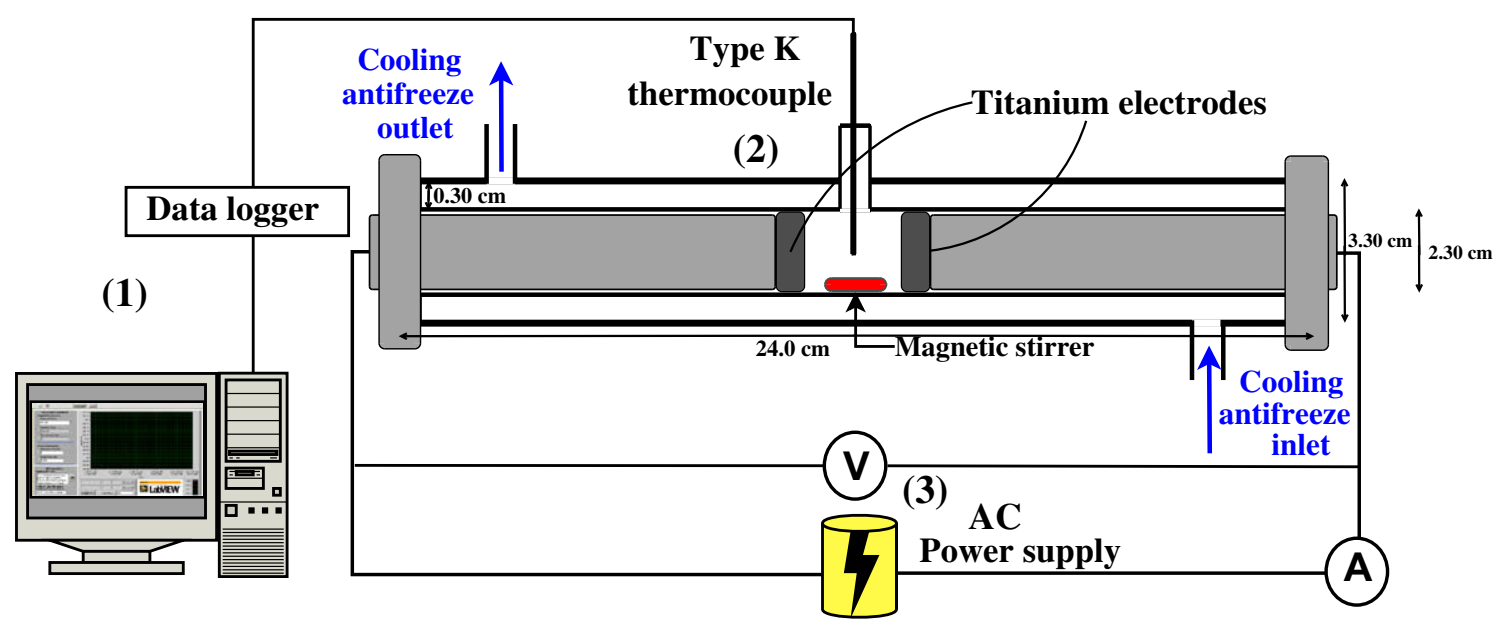

Fig. 1. MEF apparatus: (1) temperature control and data logging; (2) MEF container and (3) power supply system.

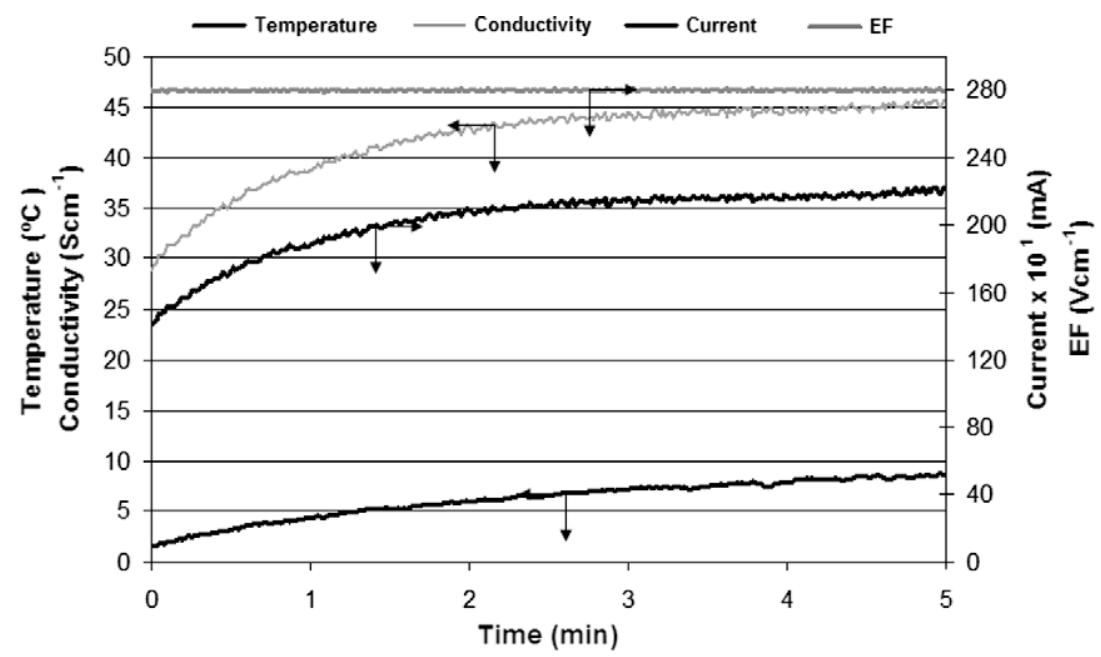

Fig. 2. Demonstrative plot of the experimental conditions: temperature, conductivity, current and electric field variations during a $280 \mathrm{~V} \mathrm{~cm}^{-1}$ treatment.

\subsection{E. coli microbial counts}

Samples containing the treated bacteria were immediately cooled in a ice bath. The sample cooled down from the work temperature (always $<25^{\circ} \mathrm{C}$ ) to approximately $3{ }^{\circ} \mathrm{C}$ in less than $20 \mathrm{~s}$. The viability was assayed by counting colony forming units (CFU). Samples of $100 \mu \mathrm{L}$ were taken both from the treated and non-treated suspensions and serially $(1: 10)$ diluted in $0.1 \%(\mathrm{w} / \mathrm{v})$ peptone (Difco, 211677, USA) water. The dilutions were plated in sixplicate, on MacConkey Agar No. 3 and incubated at $35^{\circ} \mathrm{C}$ for $24 \mathrm{~h}$, after which colonies were counted.

\subsection{Scanning electron microscopy}

Scanning electron microscopy (SEM) was used to examine the surface structure and morphology changes of the E. coli cells after the MEF inactivation treatments. For the preparation of the SEM samples the following steps were followed: the cell suspension was filtered through a $25 \mathrm{~mm}$ polycarbonate membrane filter (Whatman, Nuclepore Track-Etch Membrane, 110606, UK) with a pore size of $0.2 \mu \mathrm{m}$. Then, the membrane was placed in a $2.5 \%$ glutaraldehyde solution (Merck, 4239, Germany) that was buffered with a $0.2 \mathrm{M}$ sodium cacodylate solution (Electron Microscopy Sciences, 12300 , USA) for $2 \mathrm{~h}$. The glutaraldehyde solution was removed and the membrane was washed three times with $0.2 \mathrm{M}$ sodium cacodylate solution, and then fixed with $2 \%$ osmium tetroxide aqueous solution for $30 \mathrm{~min}$ (Electron Microscopy Sciences, 19192, USA). To remove the osmium tetroxide the membrane was rinsed with $0.2 \mathrm{M}$ sodium cacodylate solution two times. The samples were dehydrated by immersion in a series of ethanol solutions (30\%, 50\%, 70\%, 80\%, 90\%, 95\% and $100 \%(\mathrm{v} / \mathrm{v})$ ) for $15 \mathrm{~min}$. Critical point drying with liquid $\mathrm{CO}_{2}$ (Balzers Union, CPC 020, Liechtenstein) was performed on the samples before coating with gold; the samples were subsequently observed under a SEM equipment (Oxford Inca Energy 350, Jeol JSM6301F, UK).

\subsection{Model development and statistical analysis}

\subsubsection{E. coli death kinetics}

Isothermal death kinetics of microorganisms have been thoroughly described during conventional heating $(\mathrm{CH})$ to follow the Bigelow model $(D-z)$ (Leguérinel et al., 2007).

However, in the present work it is postulated that the temperature effects were replaced by those of the electric fields. As there is no previous indicator of death kinetics of any microorganism submitted to a similar treatment, a prior data exploration was necessary before proposing a kinetic model and performing the full non-linear regression analysis. This exploratory analysis was performed in three steps: (i) identification of the kinetic law valid for the different electric field curves; (ii) identification of the 
dependency of the kinetic rate constant from electric field; and (iii) full model fitting by non-linear regression. At the end of this procedure, it was possible to propose a mathematical model to describe the $E$. coli death under different electric field values during MEF treatments (Eqs. (1) and (2))

$$
\begin{aligned}
& \frac{N}{N_{0}}=\exp \left[-\frac{\ln (10)}{D_{E F_{\text {ref }}}} \cdot 10^{\frac{E F-E F_{\text {ref }}}{z_{\text {EF }}}} \cdot t+\int_{0}^{t} W(t) d t\right] \\
& D(t)=D_{c_{\text {ref }}} \cdot 10^{\left(-\frac{E F-E F_{\text {ref }}}{z_{\text {EF }}}\right)}
\end{aligned}
$$

$N$ and $N_{0}\left(\right.$ CFU ml ${ }^{-1}$ ) are the number of viable E. coli colonies at time $t(\mathrm{~min})$ and time zero, respectively, $D_{E F_{\text {ref }}}(\mathrm{min})$ is the decimal logarithmic viability decay rate, $E F$ is the electric field $\left(\mathrm{V} \mathrm{cm}^{-1}\right), E F_{\text {ref }}$ is the reference electric field $\left(\mathrm{V} \mathrm{cm}^{-1}\right), z_{E F}\left(\mathrm{~V} \mathrm{~cm}^{-1}\right)$ is the number of $\mathrm{V} \mathrm{cm}^{-1}$ required to reduce one decimal logarithmic cycle the value of $D_{E F_{\text {ref }}}$, and $W(t)$ is the error function.

The death kinetic parameters ( $D$ and $z$ ) were obtained by a onestep optimization procedure (Arabshahi and Lund, 1985), by maximizing the likelihood function with respect to the error function $W(t)$, where $W(t)$ is normally distributed with mean equal to zero and uncorrelated noise $\left(\int_{0}^{t} W(t) d t=0\right)$. The model parameters were obtained by using the Gauss-Newton search algorithm, to solve the normal equations and to obtain the parameters estimates, using as first estimates the two-step procedure kinetic estimates (Arabshahi and Lund, 1985). The parameter's variance and covariance was obtained by computing the variance-covariance matrix of the regression coefficients and model variance was estimated by the mean standard error (MSE) (Bates and Watts, 1988; Neter et al., 1996).

Kinetic models were validated by performing a randomized residuals lack of fit test (F-test) (Manly, 1997). These results were also used to compute the studentised effect ( $t$-ratio) $\left(\beta_{i} / s\left\{\beta_{i}\right\}\right)$ for each variable to determine the statistical validity of the estimated parameters. Residuals were also examined for outliers, randomness and tested for normality (Bates and Watts, 1988).

\section{Results and discussion}

\subsection{E. coli death kinetics}

MEF death kinetic data are presented in Table 1. From the direct observation of the data it is possible to conclude that the electric field strength is an extremely significant variable in terms of death rate, ranging from a very small death rate at $50 \mathrm{~V} \mathrm{~cm}^{-1}$ to significant death rate values at electric fields of $280 \mathrm{~V} \mathrm{~cm}^{-1}$.

MEF E. coli death kinetics were investigated by a two-step methodology. In the first step, the kinetic order is estimated for each electric field strength, and in the second step, a relationship between kinetic death rates and electric field strength is established. After the reaction order is established and its behavior with electric field strength determined, the one-step optimization procedure is used. A first exploration of the MEF data revealed that the $E$. coli death curves at any electric field strength follow a 1st order kinetics (Fig. 3). Further, the kinetic death rate tends to increase exponentially with the electric field, and therefore a "Bigelow-like" pattern is expected. Taking into consideration these observations, the model proposed (see Eqs. (1) and (2)) was used to describe our results.

The one-step optimization procedure presents a $D_{120 \mathrm{~V} \mathrm{~cm}^{-1}}$ of $17.581 \pm 1.301 \mathrm{~min} \quad\left(t\right.$-value $\left.=13.520 ; \quad p<2 \times 10^{-6}\right) \quad$ and $z_{\text {MEF }}=116.903 \pm 4.848\left(\mathrm{~V} \mathrm{~cm}^{-1}\right)\left(t\right.$-value $\left.=24.120 ; p<2 \times 10^{-6}\right)$, and the full model shows a SE of 1.083 and a correlation coefficient with the experimental data of 0.8374 .

Residuals analysis is presented in Fig. 3b and c; the second figure clearly shows that the semi-studentised residuals are approximately normally distributed $(p>0.90)$. Residuals do not only show a small number of outliers at $280 \mathrm{~V} \mathrm{~cm}^{-1}$, but also these small deviations are neglectable when calculating the regression error. The plot of the residuals $v s$. the normalized residuals (Fig. 3d) confirms this, as the small number of outliers is now corresponding to the small number of data points which do not fit the $y=x$ line, for values of residuals below -4 .

This model passes the lack of fit test $(p<0.001)$, which statistically ensures that $E$. coli death kinetics under the studied MEF conditions can be described by the proposed "Bigelow-like" kinetics. This can be observed in Fig. 4, which represents the values predicted by the model $v s$. the original data.

\subsection{Potential factors influencing the death kinetics of $E$. coli cells}

For the tests performed at $280 \mathrm{~V} \mathrm{~cm}^{-1}$ the samples were diluted with buffer in order to decrease their initial electrical conductivity. This was important to avoid excessive heat dissipation and the consequent excessive temperature rise during the experimental runs. Therefore, various conditions were modified that might influ-

Table 1

\begin{tabular}{|c|c|c|c|c|c|}
\hline \multirow[t]{2}{*}{ Time (min) } & \multicolumn{5}{|l|}{$\mathrm{EF}$} \\
\hline & $50 \mathrm{~V} \mathrm{~cm}^{-1}$ & $160 \mathrm{~V} \mathrm{~cm}^{-1}$ & $180 \mathrm{~V} \mathrm{~cm}^{-1}$ & $220 \mathrm{~V} \mathrm{~cm}^{-1}$ & $280 \mathrm{~V} \mathrm{~cm}^{-1}$ \\
\hline 0 & $85.027 \pm 3.108$ & $100.560 \pm 9.568$ & $92.512 \pm 11.446$ & $102.787 \pm 2.597$ & $97.791 \pm 4.794$ \\
\hline 0.5 & - & - & - & - & $68.103 \pm 12.373$ \\
\hline 1 & $84.083 \pm 2.156$ & - & $64.179 \pm 11.616$ & $93.606 \pm 8.332$ & $18.081 \pm 13.355$ \\
\hline 1.5 & - & - & - & - & $8.482 \pm 0.936$ \\
\hline 2 & $82.882 \pm 2.645$ & $68.633 \pm 2.598$ & $108.450 \pm 22.624$ & $76.628 \pm 5.155$ & $4.848 \pm 2.390$ \\
\hline 2.5 & - & - & - & - & $1.852 \pm 2.315$ \\
\hline 3 & $78.358 \pm 6.701$ & - & $52.734 \pm 8.610$ & $62.207 \pm 4.805$ & $1.084 \pm 0.964$ \\
\hline 4 & $79.821 \pm 11.230$ & $56.406 \pm 2.707$ & $51.253 \pm 14.139$ & $83.146 \pm 2.933$ & $2.377 \pm 1.053$ \\
\hline 5 & $74.356 \pm 2.454$ & - & $61.120 \pm 9.571$ & $45.819 \pm 1.720$ & - \\
\hline 6 & $81.067 \pm 3.905$ & $55.641 \pm 2.041$ & $43.311 \pm 6.640$ & $44.663 \pm 5.496$ & $0.139 \pm 0.115$ \\
\hline 7 & $100.990 \pm 21.003$ & - & $31.733 \pm 4.441$ & $37.265 \pm 8.266$ & - \\
\hline 8 & $66.542 \pm 2.176$ & $54.197 \pm 1.381$ & $14.715 \pm 1.647$ & $26.411 \pm 2.206$ & $0.136 \pm 0.142$ \\
\hline 9 & $70.619 \pm 2.711$ & - & $8.908 \pm 2.415$ & $31.666 \pm 1.925$ & - \\
\hline 10 & $61.143 \pm 6.264$ & $49.622 \pm 3.890$ & $3.291 \pm 1.941$ & $4.818 \pm 4.190$ & $0.113 \pm 0.128$ \\
\hline 12 & - & $32.964 \pm 2.688$ & - & - & - \\
\hline 14 & - & $30.381 \pm 1.314$ & - & - & - \\
\hline 16 & - & $49.868 \pm 4.595$ & - & - & - \\
\hline 18 & - & $41.618 \pm 5.438$ & - & - & - \\
\hline 20 & - & $3.751 \pm 1.703$ & - & - & - \\
\hline
\end{tabular}

E. coli death kinetics during MEF treatment (CFU ml $\left.{ }^{-1} \times 10^{4}\right)$. 


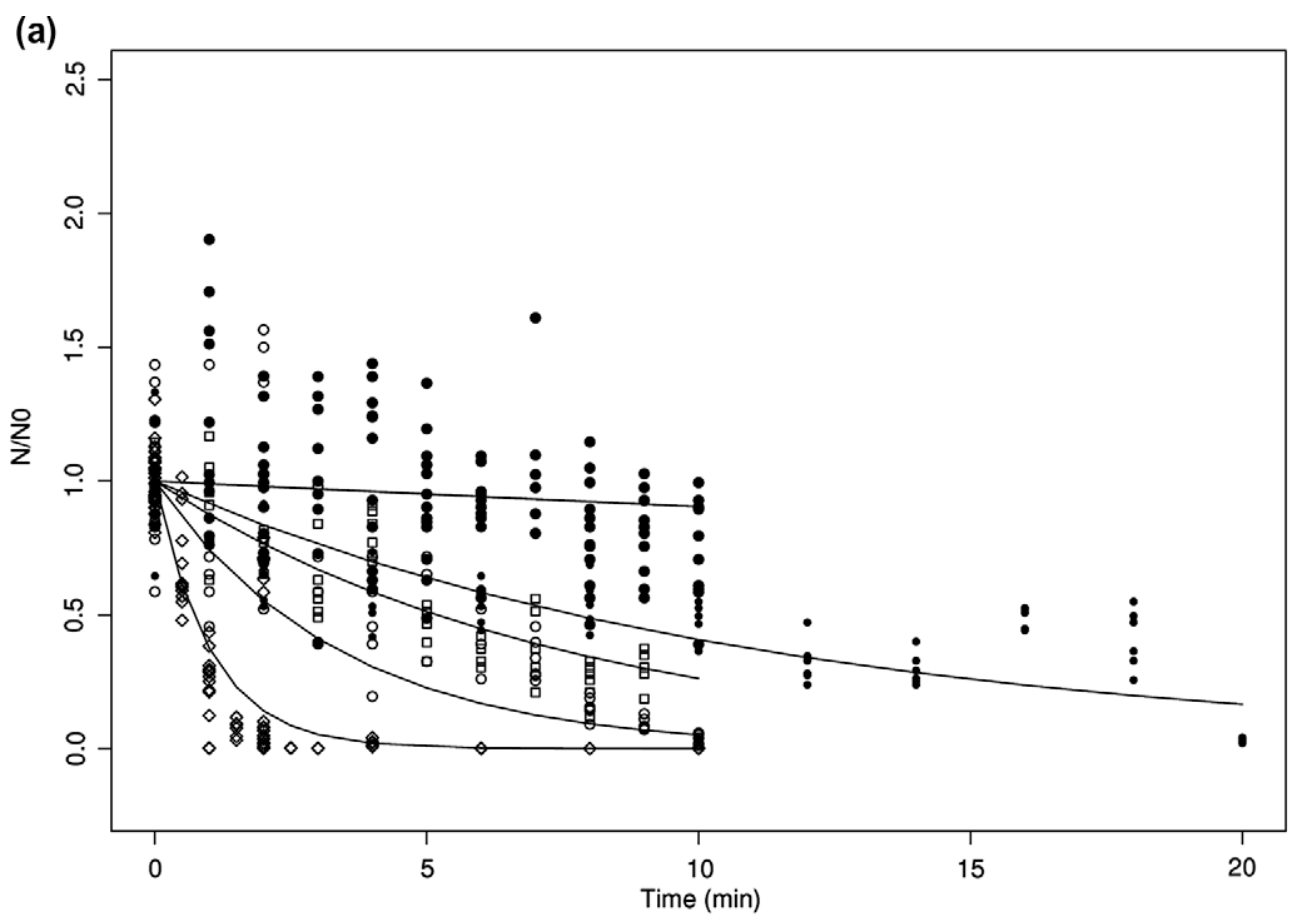

(b)

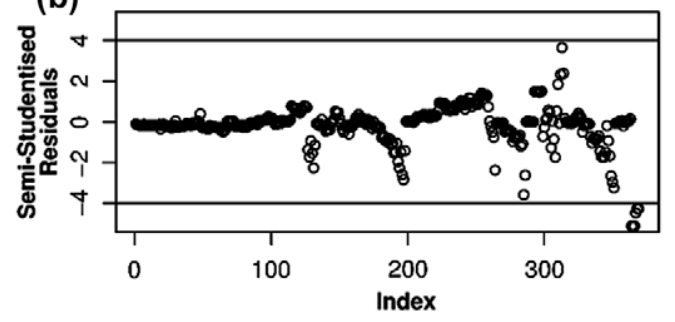

(c)

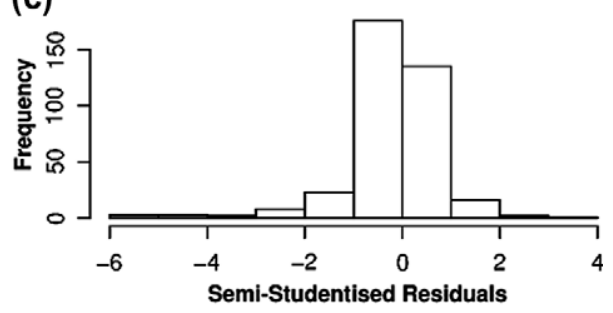

(d)

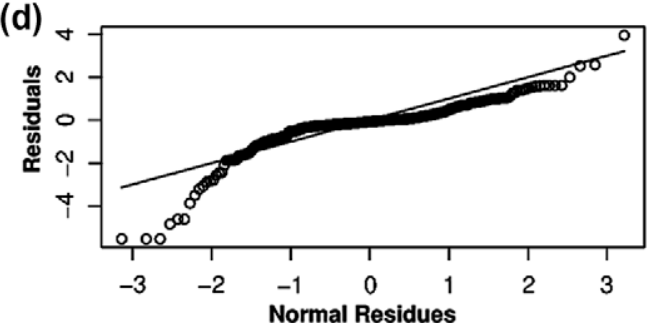

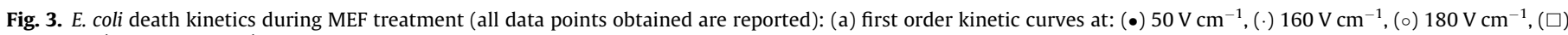

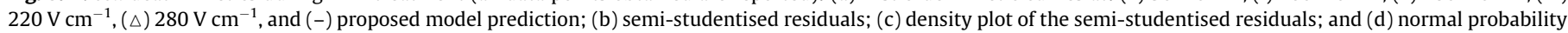
plot of residuals.

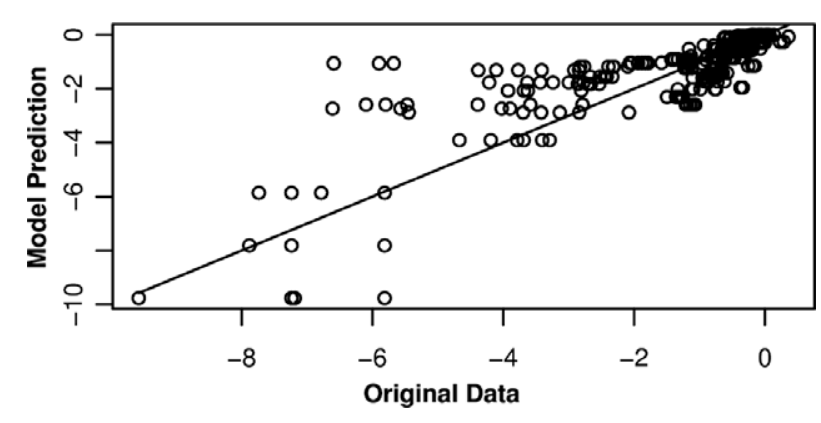

Fig. 4. Model prediction (-) vs. the original data points (o). ence the cells inactivation under the electric field. In this section the possible factors that could influence the inactivation effects are discussed.

\subsubsection{Initial cell concentration}

The initial microbial concentration may play a important role in the inactivation effects of the electric fields, however there is some controversy concerning the effects of the initial concentration on the viability loss of a cell population during inactivation treatments with electric fields. Further, most of the works dealing with inactivation effects of electric fields were performed under PEF conditions, which is not the case of the present work. Álvarez et al. (2000) reported that the initial concentration may not affect the microbial inactivation for PEF treatments, whereas there are also reports stating that the initial cell concentration is a relevant 
parameter on the microbial inactivation results (Jayaram et al., 1992; Zhang et al., 1994). It was previously reported by Zhang et al. (1994) that PEF inactivation of S. cerevisiae and E. coli was inversely correlated to initial cell concentration, assuming the hypothesis that a protection mechanism due to cluster formation occurs. The cells inside those clusters may have not been submitted to an electric field as strong as that hitting the external cells, resulting in lower inactivation. However, other authors found that the microorganisms inactivation was linearly enhanced by the increasing of the innoculum size on the PEF inactivation of S. cerevisiae (Molinari et al., 2004). Similar results were also found by Jayaram et al. (1992) on the inactivation of Lactobacillus brevis by PEF. They formulated an hypothesis based on the differences between the membrane surfaces charges due to polarization created when cells are exposed to an electric field. Consequently the cells are attracted to each other and form a "pearl chain", due to the attractive forces arising from dipoles. When the cell concentration increases, the probability of forming pearl chain increases. Therefore, the size of these "equivalent cells" increases leading to a higher efficiency of PEF treatment as a result of the lower critical electric field value for bigger microbial cells (Hülsheger et al., 1983).

\subsubsection{Medium electrical conductivity}

Certainly that the electrical conductivity has an important role in the inactivation of the microorganisms. However, in this case, when diluting the solution subjected to $280 \mathrm{~V} \mathrm{~cm}^{-1}$ the electrical conductivity has been modified (at the same temperature) to lye within the range of the values measured for the other electric fields applied, as shown in Fig. 5. This should ensure an identical influence of this parameter on the death kinetics, although the published works are not consistent when dealing with this subject. It has been previously reported that the conductivity of the treatment medium did not influence the inactivation of Salmonella senftenberg by PEF, being the electric fields strength, pulse length and the number of pulses constant (Álvarez et al., 2000). Other works reported that, for a constant energy input, an increase in conductivity of the treatment medium resulted in a decrease in inactivation in several microorganisms (Wouters et al., 1999; Vega-

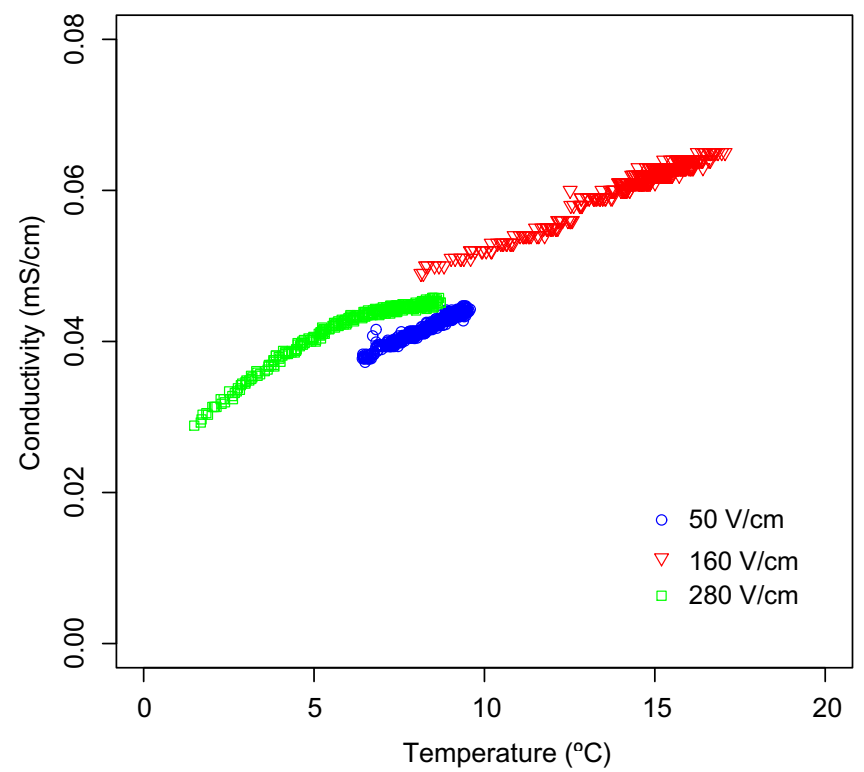

Fig. 5. Electric conductivity variation of the treatment medium (containing cells) with the temperature during exposure to: (o) $50 \mathrm{~V} \mathrm{~cm}^{-1},(\Delta) 160 \mathrm{~V} \mathrm{~cm}^{-1}$ and $(\square)$ $280 \mathrm{~V} \mathrm{~cm}^{-1}$.
Mercado et al., 1996). From this point of view, it could be concluded that it is more advantageous to work with a medium of low conductivity levels (the buffer used as treatment medium had and electrical conductivity of $6.78 \mu \mathrm{S} \mathrm{cm}^{-1}$ at $23^{\circ} \mathrm{C}$ ).

\subsection{Electric field effects on E. coli}

The effect of the medium temperature on the microbial inactivation using electric fields has been reported by Wouters et al. (2001). In the present work, the heating effects of MEF microbial inactivation kinetics were reduced to minimum by working at ambient temperature and preventing the temperature from rising during the application of the electric treatment (see Fig. 2). Therefore, the observed decrease in viable counts can be attributed to the applied electric field treatment and not to increases of temperature caused by Joule heating - the temperature of the medium did not exceed $25^{\circ} \mathrm{C}$ at any stage. Being this a nonlethal temperature, the observed effects can be designated as non-thermal (Gowrishankar et al., 2005). The mechanism underlying the inactivation of microorganisms using an electric field has not yet been fully understood. Exposure of a biological cell to an electric field can produce a variety of profound biochemical physiological responses. Most of these responses are based on the modification of the transmembrane potential by the application of an external electric field. If the field strength exceeds a certain threshold value (it can range between 0.2 and $1 \mathrm{~V}$ ), it can result in pore formation in the membrane - this phenomenon is known by electroporation or electropermeabilization. The lowest values of transmembranar potential are attained in the cells poles and are proportional to the cell diameter. Considering this, larger cells are more fragile than smaller ones and the maximal damage is caused in the cell poles. The critical potential is also dependent of the membrane structure as well as of the temperature, decreasing with temperature increase or membrane tension (Weaver and Chizmadzhev, 1996). Membranes can suffer both irreversible or reversible breakdown. Reversible breakdown generally occurs when the transmembrane potential is lower than $1 \mathrm{~V}$. If a reversible process takes place, the membrane reestablishment can occur within seconds or minutes (Simpson et al., 1999). In the present work, if cell damage was only due to reversible pore formation, treated bacteria possibly had enough time to repair, since the cells were on ice more than a few minutes until plating to determine survival of bacteria. For higher transmembrane potential, longer electric pulses, or even by the application of a high electric field, the electroporation is usually irreversible.

There are several works in the literature that present interesting results with the use of MEF. Alternated current fields of weak intensity (e.g. 20-160 $\mathrm{V} \mathrm{cm}^{-1}$ ) have been shown to change membrane conductance and successfully achieve the transfection of E. coli by plasmid DNA (Xie and Tsong, 1990). Through the application of low voltage long duration pulses $(100 \mathrm{~ms}$ pulses of $75 \mathrm{~V} \mathrm{~cm}^{-1}$ with an interval of $100 \mathrm{~ms}$ ) to human cells, it was possible to achieve electroporation using electric fields as low as $75 \mathrm{~V} \mathrm{~cm}^{-1}$ (Matsuki et al., 2008). They proposed that consecutive pulses of a voltage lower than the membrane breakdown threshold can accumulate the membrane potential, eventually until it reaches the threshold required to induce electroporation, disrupt the membrane integrity and resulting in the cell death. We expect that something similar happened with the E. coli cells when under the influence of MEF at growth temperatures. The electric field was applied for long periods on time (minimum of $2.5 \mathrm{~min}$ and maximum of 20 min for the lower voltages), enabling the accumulation of the transmembrane potential until the threshold, causing the permeabilization of the membrane and finally leading to cell death. Machado-Aranda et al. (2005) reported that using a MEF of $200 \mathrm{~V} \mathrm{~cm}^{-1}$ it was possible to achieve gene transfection in vivo 
by PEF. In other work it was observed that the application a sinusoidal, low frequency $(1-120 \mathrm{~Hz})$ and small voltage electric field $\left(3-23 \mathrm{~V} . \mathrm{cm}^{-1}\right)$ may have provoked a reorganization of the cell cytoskeletal and plasma membrane structures, providing pathways for cell surface receptors to migrate anharmonically (Cho et al., 1994).

The intensification of electric fields across cell membranes is believed to be responsible, through membrane rupture and reversible membrane breakdown processes, for certain types of tissue damage in electrical trauma cases which cannot be attributed to Joule heating (Bardos et al., 2000). Also, cell membranes can contain structural defects and are mixtures of lipids, proteins, and inclusions, all of which may reduce the threshold potential for pore formation (Partenski et al., 2007). Considering these assumptions together with the results obtained from the E. coli treatments, it is viable to consider that the application of electric fields, at moderate $\left(50-280 \mathrm{~V} \mathrm{~cm}^{-1}\right.$.) values, is feasible for the inactivation of this microorganism.

\subsection{SEM examination of MEF treated E. coli}

Because of the small size of electropores it is very difficult to obtain visual evidence (such as imaging at the membrane level). The observation of electroporated red blood cells under freezing electron microscopy revealed large pores with crater and volcano funnels forms, having diameters from $50 \mathrm{~nm}$ to $0.1 \mu \mathrm{m}$. The size of these pores most probably resulted from the enlargement of smaller primary pores by osmotic or hydrostatic pressure due to Maxwell stress (Neumann et al., 1999). Detailed observations of the E. coli cells, after SEM analyses, revealed different cell morphology after MEF treatments (Fig. 6b-d), comparing with the non-treated cells (Fig. 6a). If such differences are not too evident for the softer treatment (at $50 \mathrm{~V} \mathrm{~cm}^{-1}$, Fig. $6 \mathrm{~b}$ ), they are evident for the higher electric field values (Fig. 6c and d). The treated cell surface became rougher, indicating that the presence of the electric field provoked alterations in the membrane structure. Although these results do not allow the establishment of a direct relation between the increase of the applied electric field and the damage caused in the cell membrane, they clearly show that when subjected to electric field, the cell membranes change. When related with the results presented above concerning cell death rates, it is possible to conclude that there is a very probable connection between cell death and changes in the cell membrane morphology/functionality. Further studies are, however, needed to clarify these assumptions.

\section{Conclusions}

The work presented have clearly shown that the electric field is responsible for the non-thermal death of E. coli cells. The experimental design used has excluded the possibility of synergistic effects of temperature. It was statistically shown that the E. coli death kinetics under MEF treatment could be described by the proposed non-thermal model $(p<0.001)$. When applying electric fields to biological cells and tissues, heating is inevitable due to Joule effect. With this system we were able to prevent the temperature rise from the extracellular medium. So, the inactivation of E. coli is mainly due to the application of an electric field and can be designated as a non-thermal effect. The electric field effect becomes very significant above $160 \mathrm{~V} \mathrm{~cm}^{-1}$. Furthermore, the use of electric fields above $220 \mathrm{~V} \mathrm{~cm}^{-1}$ promotes death rates of $3 \log _{10} \mathrm{cy}$ cles of $E$. coli in less than $6 \mathrm{~min}$, and even higher rates when higher electric fields are applied, showing that it is a feasible technique for the inactivation of this microorganism and, presumably, of many others. The observation of E. coli cells after the exposure to MEF by SEM revealed changes at the cell membrane level, indicating a possible cause for the cell death. With the support of the results
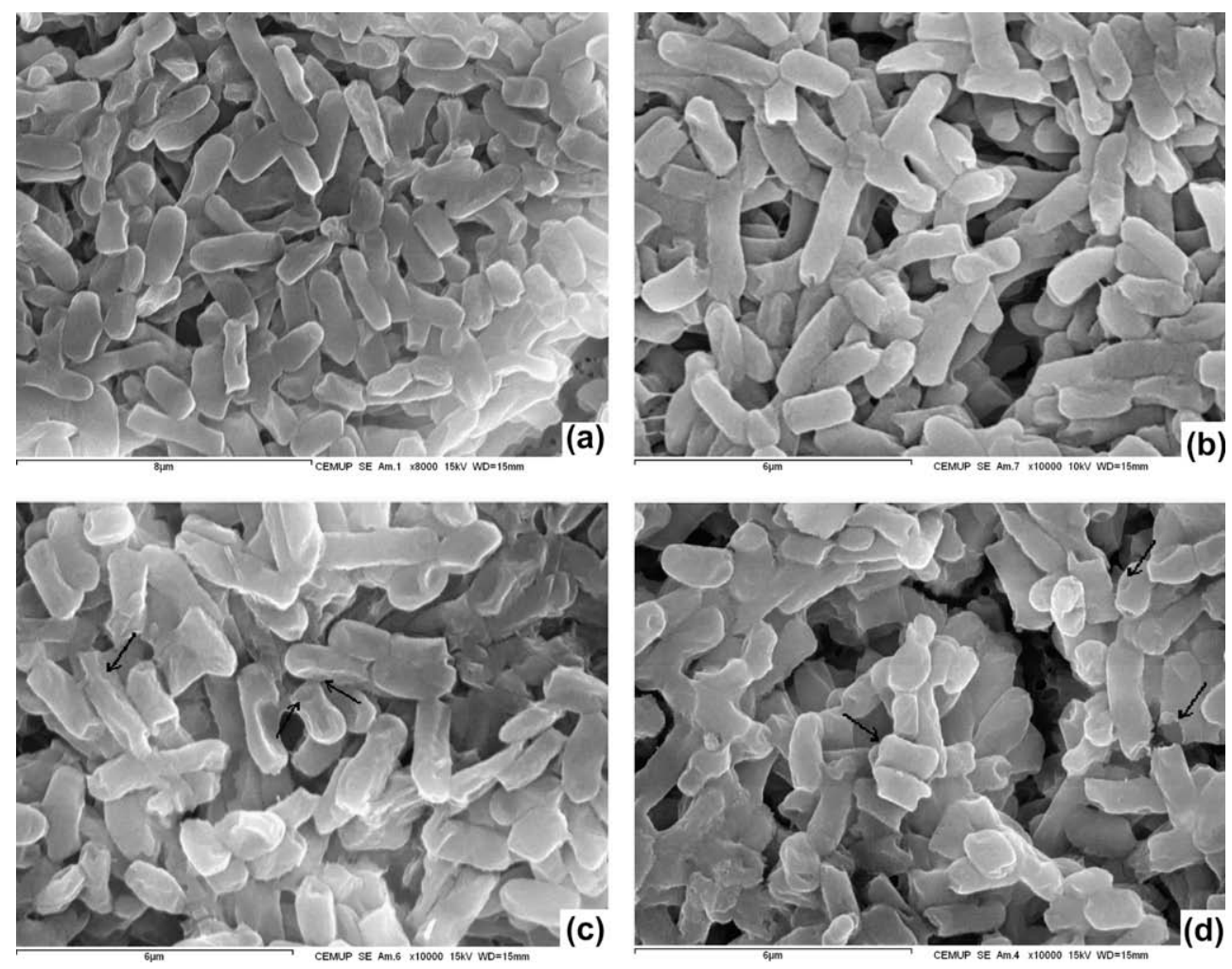

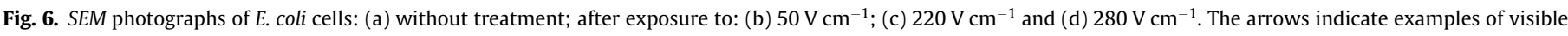
damages on the cell surface. 
presented above, MEF shows a great potential, with advantage, for sterilization of thermolabile products (e.g. serum and other physiological fluids, food products), by itself or as a complement of the traditional heat-dependent techniques. Nevertheless, there are still numerous aspects to consider before proposing MEF as the main process for microbial inactivation, for example, in the food industry that aims the production of microbiologically safe food products and to prolong the product shelf life. It is necessary a deeper understanding of the electric field effects on biological systems; to gather extensive data of electric fields effects on different microorganisms, enzymes and nutrients; and to understand the effects of food matrices on the electric field efficiency. Due to the complexity of phenomena occurring during an electric field treatment of a food product, and also the interaction between electrical parameters and food intrinsic characteristics, it is necessary to profoundly study each case before choosing a non-thermal technology using electric fields (like MEF or PEF), as the main inactivation agent of an industrial process.

\section{Acknowledgements}

The author R.C. Martins gratefully acknowledges his post-doctoral grant (SFRH/BPD/26133/2005) to the Fundação para a Ciência e Tecnologia (FCT), Portugal.

\section{References}

Álvarez, I., Raso, J., Palop, A., Sala, F.J., 2000. Influence of different factors on the inactivation of Salmonella senftenberg by pulsed electric fields. International Journal of Food Microbiology 55, 143-146.

Anderson, A., Finkelstein, R., 1919. study of the electro pure process of treating milk Journal of Dairy Science 2, 374-406.

Arabshahi, A., Lund, D.B., 1985. Considerations in calculating kinetics parameters from experimental data. Journal of Food Process Engineering 7 (4), 239-251.

Aronsson, K., Ronner, U., Borch, E., 2005. Inactivation of Escherichia coli, Listeria innocua and Saccharomyces cerevisiae in relation to membrane permeabilization and subsequent leakage of intracellular compounds due to pulsed electric field processing. International Journal of Food Microbiology 99, 19-32.

Bardos, D.C., Thompson, C.J., Yang, Y.S., Joyner, K.H., 2000. Nonlinear cell response to strong electric fields. Physics in Medicine and Biology 45, 1965-1988.

Bates, D.M., Watts, D.G., 1988. Nonlinear Regression Analysis and Its Applications. John Wiley and Sons.

Cho, M.R., Thatte, H.S., Lee, R.C., Golan, D.E., 1994. Induced redistribution of cell surface receptors by alternating current electric fields. The Journal of the Federation of American Societies for Experimental Biology 8, 771-776.

Cho, H.Y., Yousef, A., Sastry, S.K., 1999. Kinetics of inactivation of Bacillus subtilis spores by continuous or intermittent ohmic and conventional heating. Biotechnology and Bioengineering 62 (3), 368-372.

Gowrishankar, T.R., Stewart, D.A., Weaver, J.C., 2005. Model of a confined spherical cell in uniform and heterogeneous applied electric fields. Bioelectrochemistry 68, 185-194.

Ho, S., Mittal, G.S., 2000. High voltage pulsed electric field for liquid food pasteurization. Food Reviews International 16 (4), 395-434.

Hülsheger, H., Potel, J., Niemann, E.G., 1983. Electric field effects on bacteria and yeast cells. Radiation and Environmental Biophysics 22, 149-162.
Jayaram, S., Castle, G.S.P., Margaritis, A., 1992. Kinetics of sterilization of Lactobacillus brevis by the application of high voltage pulses. Biotechnology and Bioengineering 40 (11), 1412-1420.

Kulshestha, S., Sastry, S.K., 2003. Frequency and voltage effects on enhanced diffusion during moderate electric field (mef) treatment. Innovative Food Science and Emerging Technologies 4, 189-194.

Lee, C.H., Yoon, S.W., 1999, IFT ed. Effect of Ohmic Heating on the Structure and Permeability of the Cell Membrane of Saccharomyces cerevisiae, 79(B-6) Institute of Food Technologists, Chicago.

Leguérinel, I., Couvert, O., Mafart, P., 2007. Modelling the influence of the sporulation temperature upon the bacterial spore resistance, application to heating process calculation. International Journal of Food Microbiology 114, 100-104.

Machado-Aranda, D., Adir, Y., Yong, J.L., 2005. Gene transfer of $\mathrm{na}^{+}, \mathrm{k}^{+}$-atpase $\beta 1$ subunit using electroporation increases lung liquid clearance. American Journal of Respiratory and Critical Care Medicine 171, 204-211.

Machado, L.F., Vicente, A.A., Teixeira, J.A., Miranda, F., 2007. Pilot scale ohmic heating treatment of fruit puree containing particles - influence of the processing temperature on quality parameters. Engenharia Química 5, 30-36.

Manly, B.F.J., 1997. Randomization, Bootstrap and Monte Carlo Methods in Biology, second ed. Chapman and Hall, London.

Matsuki, N., Ishikawa, T., Imai, Y., Yamaguchi, T., 2008. Low voltage pulses can induce apoptosis. Cancer Letters 269, 93-100.

Molinari, P., Pilosof, A., Jagus, R.J., 2004. Effect of growth phase and inoculum size on the inactivation of Saccharomyces cerevisiae in fruit juices, by pulsed electric fields. Food Research International 37, 793-798.

Neter, J., Kutner, M.H., Nachtsheim, C.J., Wasserman, W., 1996. Applied Linear Statistical Models. Irwin

Neumann, E., Kakorin, S., Toensing, K., 1999. Fundamentals of electroporative delivery of drugs and genes. Bioelectrochemistry and Bioenergetics 48, 3-16.

Partenski, M.B., Miloshevsky, G.V., Jordan, P.C., 2007. The theoretical challenge posed by low-voltage membrane electroporation, viewed from the perspective of continuum and molecular-level models. Israel Journal of Chemistry 47, 385396.

Rastogi, N.K., 2003. Application of high-intensity pulsed electric fields on food processing. Food Reviews International 19 (3), 229-251.

Sastry, S., Yousef, A., Cho, H.Y., Unal, R., Salengke, S., Wang, W., Lima, M., Kulshrestha, S., Wongsa-Ngasri, P., Sensoy, I., 2002. Engineering and Food for the 21 Century. Chapter Ohmic Heating and Moderate Electric Field (MEF) Processing. CRC Press LLC.

Simpson, R.K., Whittington, R., Earnshaw, R.G., Russell, N.J., 1999. Pulsed high electric field causes 'all or nothing' membrane damage in Listeria monocytogenes and Salmonella typhimurium, but membrane $\mathrm{H}^{+}$-ATPase is not a primary target. International Journal of Food Microbiology 48, 1-10.

Vega-Mercado, H., Pothakamury, U.R., Chang, F.J., Barbosa-Cánovas, G.V., Swanson, B.G., 1996. Inactivation of Escherichia coli by combining ph, ionic strength and pulsed electric fields hurdles. Food Research International 29 (2), 117-121.

Vicente, A.A., Castro, I., Teixeira, J.A., 2006. Thermal Food Processing: New Technologies and Quality Issues. Chapter Ohmic Heating for Food Processing. CRC Press, Taylor \& Francis Group.

Weaver, J.C., Chizmadzhev, Y.A., 1996. Theory of electroporation: a review. Bioelectrochemistry and Bioenergetics 41, 135-160.

Wouters, P.C., Dutreux, N., Smelt, J.P.P.M., Lelieveld, H.L.M., 1999. Effects of pulsed electric fields on inactivation kinetics of Listeria innocua. Applied and Environmental Microbiology 65 (12), 5364-5371.

Wouters, P.C., Álvarez, I., Raso, J., 2001. Critical factors determining inactivation kinetics by pulsed electric field food processing. Trends in Food Science and Technology 12, 112-121.

Xie, T.D., Tsong, T.Y., 1990. Study of mechanism of electric field-induced DNA transfection. II: Transfection by low-amplitude, low-frequency alternating electric fields. Biophysical Journal 58, 897-903.

Zhang, Q.H., Monsalve-Gonzalez, A., Barbosa-Cánovas, G.V., Swanson, B.G., 1994 Inactivation of $E$. coli and $S$. cerevisiae by pulsed electric fields under controlled temperature conditions. Transactions of the ASAE 37, 581-587. 